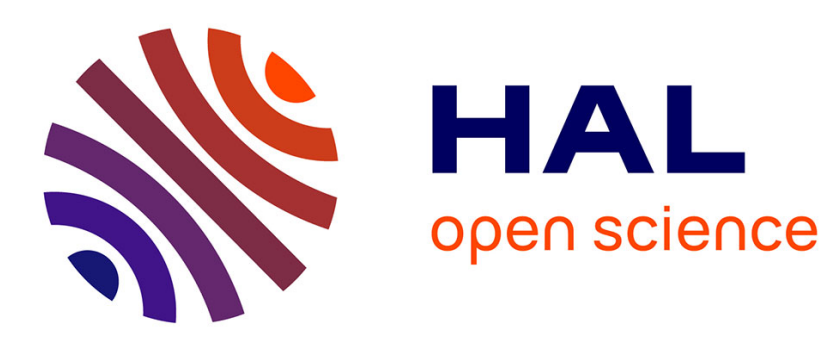

\title{
Towards efficient simulation of marked point process models for boat extraction from high resolution optical remotely sensed images \\ Paula Craciun, Josiane Zerubia
}

\section{- To cite this version:}

Paula Craciun, Josiane Zerubia. Towards efficient simulation of marked point process models for boat extraction from high resolution optical remotely sensed images. IEEE IGARSS - International Geoscience and Remote Sensing Symposium, Jul 2014, Quebec, Canada. hal-01016874

\section{HAL Id: hal-01016874 \\ https://hal.inria.fr/hal-01016874}

Submitted on 1 Jul 2014

HAL is a multi-disciplinary open access archive for the deposit and dissemination of scientific research documents, whether they are published or not. The documents may come from teaching and research institutions in France or abroad, or from public or private research centers.
L'archive ouverte pluridisciplinaire HAL, est destinée au dépôt et à la diffusion de documents scientifiques de niveau recherche, publiés ou non, émanant des établissements d'enseignement et de recherche français ou étrangers, des laboratoires publics ou privés. 


\title{
TOWARDS EFFICIENT SIMULATION OF MARKED POINT PROCESS MODELS FOR BOAT EXTRACTION FROM HIGH RESOLUTION OPTICAL REMOTELY SENSED IMAGES
}

\author{
Paula Craciun, Josiane Zerubia* \\ INRIA, AYIN research group \\ 2004 Route des Lucioles, BP 93, 06902 Sophia-Antipolis Cedex (France) \\ e-mail: Firstname.Lastname@inria.fr
}

\begin{abstract}
Marked point processes have proven their efficiency in solving object extraction problems from high resolution optical images. However, these complex mathematical models are difficult to simulate which usually results in high computation times. A new parallel sampler has been recently developed. Nevertheless, this sampler does not yield good results in the presence of large objects. We propose modifications to the original parallel sampler to cope with such situations. Furthermore, we implement an additional mask to increase the speedup in the case of boat extraction.
\end{abstract}

Index Terms- remote sensing, object extraction, marked point processes, high performance computing.

\section{INTRODUCTION}

Marked point process (MPP) models have been successfully applied to object extraction in high resolution remotely sensed optical images. Applications range from flamingos and boats counting to road or building extraction [1]. The configuration of objects in an image can be viewed as a realization of a MPP. A complex probability density function is defined to describe both the interactions between the objects and the likelihood of such a configuration with respect to the considered image. To find the best configuration, an optimization problem has to be solved by means of sampling algorithms such as Reversible Jump MCMC (RJMCMC) [2]. The main ideea of RJMCMC resides in the current configuration being iteratively perturbed by a proposition kernel while an acceptance ratio is being computed for the new configuration. Nevertheless, RJMCMC poses a computational burden due to its sequential nature which results in high computation times to reach convergence.

Several attempts have been made to improve the optimization procedure. A first improvement came with the implementation of Multiple Birth and Death (MBD) [3], an optimization method which allows for multiple perturbations to be performed simultaneously. This algorithm has been further

\footnotetext{
${ }^{*}$ The authors thank Astrium-EADS for providing the data and for the partial funding of this research.
}

refined to Multiple Birth and Cut (MBC) [4] which combines the advantages of MBD with those of the Graph-Cut algorithm. The most important advantage of MBC was the reduction of the number of parameters, thus resulting in an increased convergence speed. A major breakthough came with the implementation of the first parallel version of RJMCMC [5]. In spite of its sequential nature, RJMCMC has been successfully parallelized by splitting the search space in smaller cells and performing the optimization locally and concurrently in each of them. This approach resulted in an efficient sampler that yielded small computation times and brought back the focus on such optimization methods which were previously considered too slow for real applications. However, the parallel implementation on GPU presented in [5] does not take into account important considerations such as large objects located at the intersection of neighboring cells, which results in the need of inter-process communication or access to shared memory. In this paper we propose modifications to the initial sampler to account for such situations. Furthermore, we develop a fast and efficient method to discriminate between water and land areas. This latter attempt is motivated by our interest in boat detection. This paper is organized as follows: we start with a brief introduction of the general MPP framework in section 2 Section 3 describes the water/land discrimination method. Section 4 presents the weaknesses of the current parallel sampler described in [5] and contains the modifications brought to it. Results are presented in section 5 and finally conclusions are drawn in section 6

\section{MARKED POINT PROCESS MODEL FOR BOAT EXTRACTION}

We consider a MPP of ellipses. When seen from above, i.e. satellite images at nadir, the boats tend to have an elliptical shape. The object space $\mathcal{W}$, is a bounded set in $\mathbb{R}^{5}$ :

$$
\mathcal{W}=\left[0, X_{M}\right] \times\left[0, Y_{M}\right] \times\left[a_{m}, a_{M}\right] \times\left[b_{m}, b_{M}\right] \times[0, \pi]
$$

where $X_{M}$ and $Y_{M}$ are the width and height of the input image, respectively, $a_{m}$ and $a_{M}$ are the minimum and the maximum of the semi-major axis of the ellipse, $b_{m}$ and $b_{M}$ are 
the minimum and the maximum of the semi-minor axis of the ellipse and $\omega \in[0, \pi]$ is the orientation of the ellipse.

We are interested in a particular family of MPPs, namely the Gibbs processes [6]. Denoting the observed image with $y$, the density of the considered MPP is given by:

$$
f_{\theta}(X=\mathbf{x} \mid \mathbf{y})=\frac{1}{c(\theta \mid \mathbf{y})} \exp ^{-U_{\theta}(\mathbf{x}, \mathbf{y})}
$$

where $c(\theta \mid \mathbf{y})$ is a normalizing function given by:

$$
c(\theta \mid \mathbf{y})=\int_{\Omega} \exp ^{-U_{\theta}(\mathbf{x}, \mathbf{y}) \mu(d \mathbf{x})} .
$$

Here, $\theta$ is a parameter vector which allows the model to be flexible and fit different images. It has to be adjusted according to the given image. $\mu(\cdot)$ is the intensity measure of the reference Poisson process, $U_{\theta}(\mathbf{x}, \mathbf{y})$ is called the energy, $\Omega$ is the configuration space and $\mathbf{x}$ denotes the object configuration.

Using the Maximum A Posteriori (MAP) criterion, the most likely configuration which allows the extraction of objects corresponds to the global minimum of the total energy $U_{\theta}(\mathbf{x}, \mathbf{y})$ :

$$
x \in \underset{\mathbf{x} \in \Omega}{\operatorname{Argmax}} f_{\theta}(X=\mathbf{x} \mid \mathbf{y})=\operatorname{Argmin}\left[U_{\mathbf{x} \in \Omega}[\mathbf{x} \mid \mathbf{y})\right] .
$$

Parameter estimation techniques are presented in detail in [7, 8]. Once the parameter vector $\theta$ is determined, a solution to the resulting optimization problem has to be found. For the extraction of boats outside the harbor area we use the same model presented in [3] for flamingo extraction. In the case of harbor images, details on the model used are presented in [9].

\section{WATER/LAND DISCRIMINATION}

A first step to improve the performance of this framework is to limit the search for objects to the area where they are expected to appear. Since our main interest is to extract boats, it is meaningful to search for them only in the areas where water is present. This motivates our attempt to discriminate between water and land area. The water area can be clearly
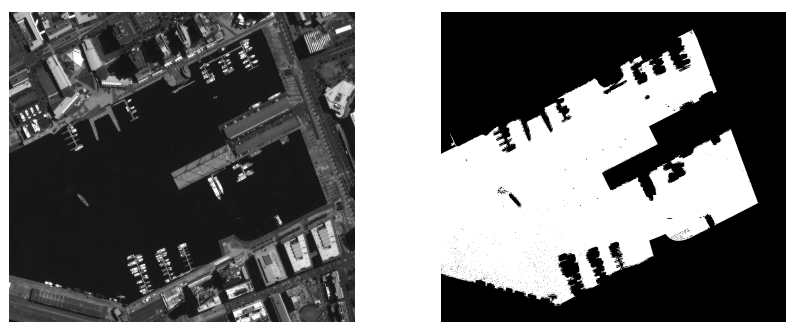

Fig. 1. left: Harbor image (c)Astrium-EADS; right: Identified water area

identified as a large area of low radiometric values. Nevertheless, the shadows of tall buildings within cities exhibit similar characteristics, as shown in Figure 1 (left). The difficulty lies in finding appropriate features to distinguish between these two area types.

In this paper we focus on a simple approach to make the separation between water and shadows. We are interested in extracting the water area under the condition that the number of misdetection should be zero, whereas the number of false alarms should be minimal. Given the low radiometric values of water, a threshold is determined by means of bimodal histogram splitting. This approach is motivated by our interest to distinguish between water and non-water area. This will identify areas of low radiometric values, which include both water and shadows. Two main features can be used to differentiate

\begin{tabular}{cccc}
\hline $\begin{array}{c}\text { Component } \\
\text { type }\end{array}$ & Size & Mean & Variance \\
\hline Shadow 1 & 8641 & 12.8962 & 1.03389 \\
Shadow 2 & 8211 & 13.0898 & 1.22846 \\
Shadow 3 & 9986 & 13.0068 & 1.00696 \\
Water & 969675 & 13.2784 & 0.377285 \\
\hline
\end{tabular}

Table 1. Comparison of characteristics of water and shadow components

between water and shadows: size of the area and radiometric variance within it. Table 1 shows the difference in feature values for the two types of areas. Based on the results presented in Table 1. we can devise an algorithm to extract the water area, similar to the one presented in [10]. The main difference is our emphasis on size. Thus, after we compute the size and the radiometric variance for each connected component identified in the threshold image, we retain only those components that have both large size and low radiometric variance. Figure 1 (right) presents the results obtained. We can observe that the water area has been correctly and entirely identified. For the purpose of boat extraction, we erode the obtained image with a circular structuring element, twice the size of the boats we are interested in extracting.

\section{HIGH PERFORMANCE COMPUTING}

The first major step towards a time-efficient approach to simulate MPPs came with the development of the parallel sampler devised in [5]. The main idea in the $2 D$ case is to divide the search space using a quadtree $\mathcal{K}$. The cells of the quadtree are divided into 4 independent sets called mic-sets and denoted $S_{m i c}$. At each iteration, one mic-set is selected and the optimization step is performed in parallel within all the cells, $c$, contained in it. The general proposition kernel $\mathcal{Q}$ is formulated as a mixture of uniform sub-kernels $\mathcal{Q}_{c, \gamma}$, where $\gamma \in \Gamma=\{$ birth and death, translation, rotation, scale $\}$. The probability of each proposition kernel is computed as $q_{c, t}=\frac{\operatorname{Pr}(\gamma)}{\# \text { cells in } \mathcal{K}}$. The computational efficiency of the sam- 


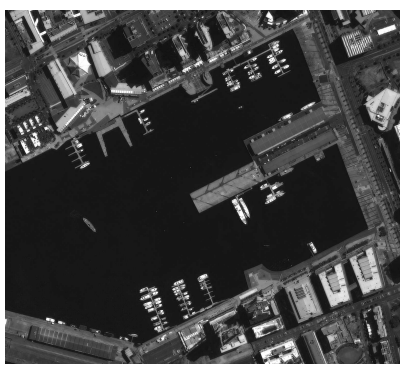

(a)

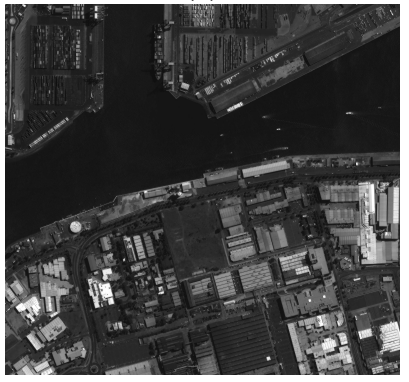

(e)

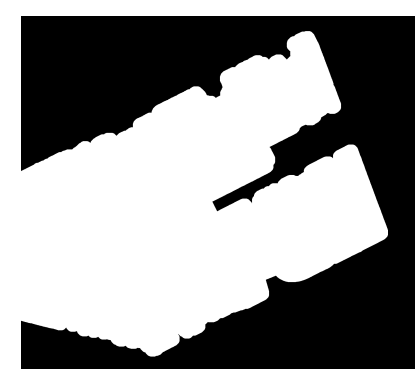

(b)

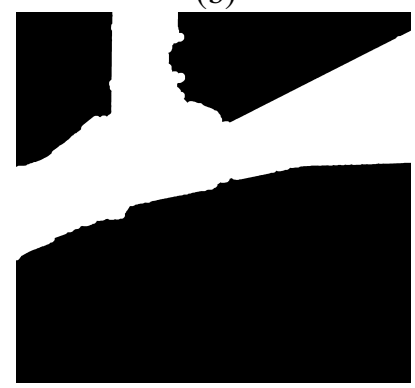

(f)

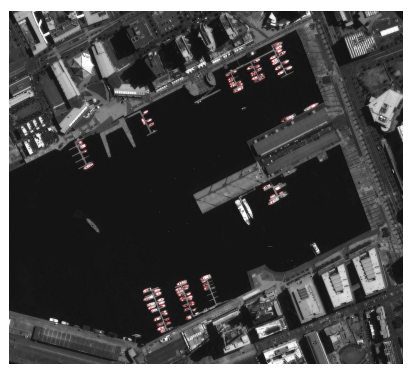

(c)

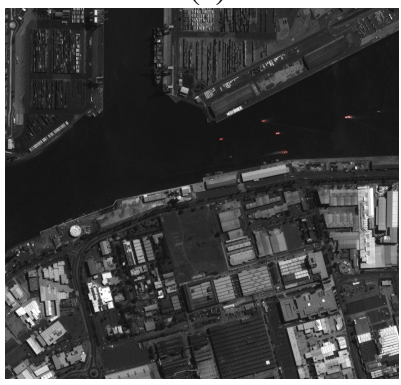

(g)

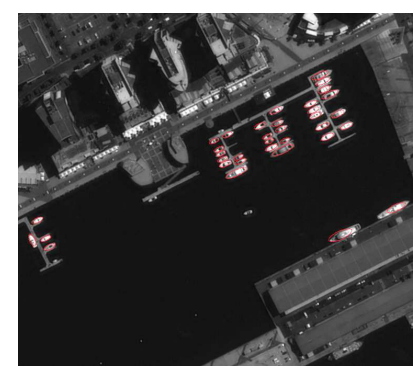

(d)

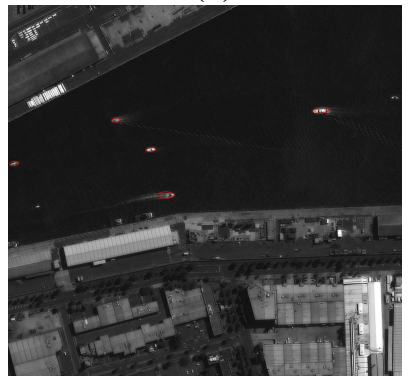

(h)

Fig. 3. (a), (e) Image of boats in and outside a harbor (c)Astrium-EADS; (b),(f) Water/Land discrimination results; (c),(g) Extraction results; (d),(h) Close-ups on extraction results.

pler has been proven for a large number of applications. The sampler in [5] makes use of GPU computing to perform the optimization. GPU computing has confirmed its usefulness in the case of data-parallel processing. In other words, if there is no need for individual processors to communicate and share data between each other, nor to access shared memory, GPU computing outstands multi-core parallelization approaches.

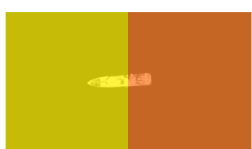

(a)

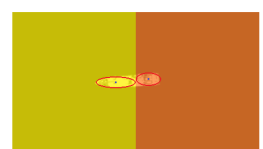

(b)

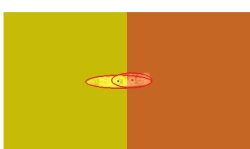

(c)
Fig. 2. (a) A large boat split in two due to the space partitioning; (b), (c) Errors which arise when processing cells independently of each other

However, this is not our case. One object can be contained in two neighboring cells, as shown in Figure 2(a). Imposing data-parallel processing in each cell independently results in two possible types of detection errors:

1. The object is split at cell boundary and thus, two smaller objects are detected (see Figure 2(b));

2. Ellipses are allowed to cross the boundary of a cell but this approach leads to several detections of the same object (see Figure 2(c))

To overcome this problem, we implement a multi-core version of the sampler with a shared memory between the cores.

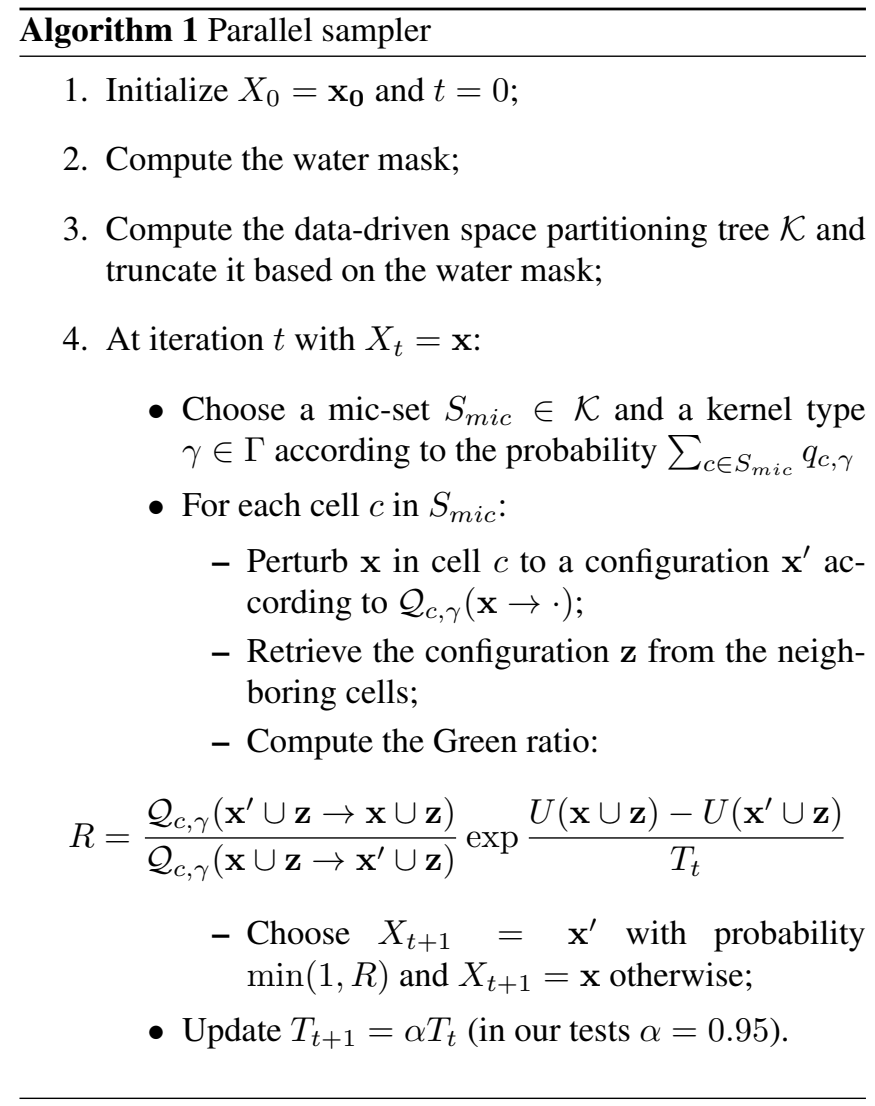

At each iteration, for each cell in a chosen mic-set $S_{m i c}$, the configurations within the neighboring cells are taken into con- 


\begin{tabular}{ccccc}
\hline \multirow{2}{*}{ \# CPU's } & \multicolumn{2}{c}{ Computation times for Figure 3 (a) } & \multicolumn{2}{c}{ Computation times for Figure 3 (d) } \\
\cline { 2 - 5 } & Water mask & No water mask & Water mask & No water mask \\
\hline 1 & $1 \mathrm{~h} 38 \mathrm{~min} 57 \mathrm{sec}$ & $2 \mathrm{~h} 4 \mathrm{~min} 5 \mathrm{sec}$ & $1 \mathrm{~h} 27 \mathrm{~min} 48 \mathrm{sec}$ & $2 \mathrm{~h} 9 \mathrm{~min} 35 \mathrm{sec}$ \\
2 & $1 \mathrm{~h} 20 \mathrm{~min} 23 \mathrm{sec}$ & $1 \mathrm{~h} 45 \mathrm{~min} 41 \mathrm{sec}$ & $33 \mathrm{~min} 43 \mathrm{sec}$ & $40 \mathrm{~min} 21 \mathrm{sec}$ \\
4 & $53 \mathrm{~min} 37 \mathrm{sec}$ & $1 h 01 \mathrm{~min} 12 \mathrm{sec}$ & $11 \mathrm{~min} 29 \mathrm{sec}$ & $15 \mathrm{~min} 26 \mathrm{sec}$ \\
8 & $44 \mathrm{~min} 7 \mathrm{sec}$ & $49 \mathrm{~min} 23 \mathrm{sec}$ & $4 \mathrm{~min} 59 \mathrm{sec}$ & $5 \mathrm{~min} 46 \mathrm{sec}$ \\
16 & $20 \mathrm{~min} 19 \mathrm{sec}$ & $23 \mathrm{~min} 1 \mathrm{sec}$ & $3 \mathrm{~min} 53 \mathrm{sec}$ & $4 \mathrm{~min} 31 \mathrm{sec}$ \\
24 & $9 \mathrm{~min} 41 \mathrm{sec}$ & $12 \mathrm{~min} 54 \mathrm{sec}$ & $2 \mathrm{~min} 32 \mathrm{sec}$ & $3 \mathrm{~min} 8 \mathrm{sec}$ \\
\hline
\end{tabular}

Table 2. Comparison of characteristics of water and shadow components

sideration. Note that this does not give rise to synchronization problems when accessing the shared memory since processors only read information in the neighboring cells and do not modify it. The modified parallel sampler is detailed in Algorithm 1

\section{RESULTS AND DISCUSSIONS}

Figure 3 presents the extraction results. Figure 3 (a) of size $1620 \times 1450$ represents an image of a harbor with a large number of objects, while Figure 3 (e) of size $2862 \times 2676$ represents a large area outside of a harbor with only a few boats. Figures 3 (b) and (f) show the area used for boat extraction after identifying the water area.

A multi-core computer with 18 Intel Xeon(R) $2.30 \mathrm{GHz}$ physical CPU's and 6 virtual CPU's was used for the computations. The computation time needed for each of the two images with respect to the number of CPU's and the use of the water mask are presented in Table 2 A birth map was considered for each extraction, independent of the existence of the water mask. As expected, the extraction times drop considerably with the increase in the number of CPU's. Results show that an increase in the number of objects present in the scene translates into a considerable increase in computation time. Finally, the usefulness of the water mask is proven by an additional decrease in computation time in both cases.

\section{CONCLUSIONS AND FUTURE WORK}

In this paper, we have presented two approaches for increasing the efficiency of simulating marked point process models for boat extraction. We discriminated between water and land area to reduce the search space. Furthermore, we have modified the parallel sampler devised in [5] to cope with large objects and implemented it on a multi-core computer with shared memory access between the processors. This approach was motivated by the necessity to access the configurations in the neighborng cells. This resulted in improved extraction results and low computation times. Future work will include an extension of this framework to time series.

\section{REFERENCES}

[1] X. Descombes, F. Chatelain, F. Lafarge, C. Lantuejoul, C. Mallet, M. Minlos, M. Schmitt, M. Sigelle, R. Stoica, and E. Zhizhina, Stochastic Geometry for Image Analysis, John Wiley and Sons, 2011.

[2] P. Green, "Reversible jump Markov Chain Monte Carlo computation and Bayesian model determination," Biometrika, vol. 82, no. 4, pp. 711-732, 1995.

[3] S. Descamps, X. Descombes, A. Bèchet, and J. Zerubia, "Automatic flamingo detection using multiple birth and death process," Proc.of ICASSP, 2008.

[4] A. Gamal-Eldin, X. Descombes, and J. Zerubia, "Multiple birth and cut algorithm for point process optimization," Proc.SITIS, pp. 35-42, 2010.

[5] Y. Verdié and F. Lafarge, "Efficient Monte Carlo sampler for detecting parametric objects in large scenes," Proc. ECCV 2012, vol. 7574, pp. 539-552, 2012.

[6] D. Stoyan, W. S. Kendall, and J. Mecke, Stochastic Geometry and its Applications, John Wiley and Sons, 1987.

[7] F. Chatelain, X. Descombes, and J. Zerubia, "Parameter estimation for a marked point process. application to object extraction from remote sensing images," Proc. EMMCVPR, vol. 5681, pp. 221-234, 2009.

[8] S. Ben Hadj, F. Chatelain, X. Descombes, and J. Zerubia, "Parameter estimation for a marked point process within a framework of multidimensional shape extraction from remote sensing images," Proc. of ISPRS Conference on Photogrammetry Computer Vision and Image Analysis, vol. XXXVIII, 2010.

[9] P. Craciun and J. Zerubia, "Unsupervised marked point process model for boat extraction in harbors from high resolution optical remotely sensed image," Proc. ICIP, 2013.

[10] P. M. Dare, "Shadow analysis in high-resolution satellite imagery of urban areas," Photogrammetric Engineering and Remote Sensing, vol. 71, pp. 169-177, 2005. 